AUTHOR CORRECTION

\title{
Author Correction: Mechanical activation of spike fosters SARS- CoV-2 infection
}

Wei Hu, Yong Zhang, Panyu Fei, Tongtong Zhang, Danmei Yao, Yufei Gao, Jia Liu, Hui Chen, Qiao Lu, Tenny Mudianto, Xinrui Zhang, Chuxuan Xiao, Yang Ye, Qiming Sun (D), Jing Zhang, Qi Xie, Pei-Hui Wang, Jun Wang, Zhenhai Li, Jizhong Lou (iD) and Wei Chen (iD

(C) CEMCS, CAS 2021

Cell Research (2021) 31:1223; https://doi.org/10.1038/s41422-021-00576-9

Correction to: Cell Research https://doi.org/10.1038/s41422-02100558-x, published online 31 August 2021

In the initial published version of this article, there was an error in the first affiliation. The correct first affiliation is "Department of Cell
Biology and Department of Cardiology of the Second Affiliated Hospital, Zhejiang University School of Medicine, Hangzhou, Zhejiang, China". This correction does not affect the description of the results or the conclusions of this work. 